NON INVASIVE CARDIOLOGY 


\title{
First 100 Dobutamine Stress Echocardiography in Medicare National Hospital, Nepal
}

\author{
Dr, Abani B, Upadhyaya, Dr. Denis Shrestha,
}

\section{Background:}

In patients with known or suspected CAD, diagnosis and risk stratification can be aided by non-invasive tests for myocardial ischemia. The scientific use of stress echo, started twenty years ago and for at least a decade, it has been considered a method of established usefulness. Medicare National Hospital, Kathmandu introduced stress echo, in June 2003 and has performed over 140 stress echo. so far, out of which 130 are dobutamine stress echo. The data of first 100-dobutamine-stress echo. were analyzed and presented.

\section{Methods and Results}

Dobutamine was Infused intravenous at rates of $10-20-30$ and $40 \mathrm{microgm} / \mathrm{kg} / \mathrm{min}$ with 3 minutes duration in each stage and multiple two-dimensional echocardiographic images of the heart were obtained from the parasternal long and short-axis and the apical fourchamber and two-chamber views at each stage...Appearance of new myocardial wall motion abnormality was reported as positive for CAD. The indication for dobutamine stress echocardiography were suspected CAD, risk stratification following myocardial infarction and evaluation of patients with known CAD before noncardiac surgery and the assessment of myocardial viability in patients with resting myocardial wall motion abnormality, $81 \%$ of patients were hypertensive, $21 \%$ had diabetes mellitus, $37 \%$ had dyslipidemia and $48 \%$ had 2 or more risk factors. Three-forth of the patients were males and most of the patients were in their fifth decade of life, 30 patients out of 100 had positive test for inducible myocardial ischemia. Of all patients with positive results 24 were hypertensive, 7 had diabetes mellitus, 11 were dyslipidemic and 14 had two or more risk factors. Out of 66 patients with normal stress ECG, 8 had positive results for myocardial ischemia detected by echocardiography and of 34 patients with abnormal stress ECG 12 patients didn't have wall motion abnormality in echocardiography, thus reported as negative. No significant differences were seen in correlation of stress ECG and echocardiography between different gender. There were no major complications. 


\section{Conclusion:}

Of all stress tests for evaluation of patients with known or suspected CAD Dobutamine stress imaging is an useful alternative, safe and superior for patients with: abnormal baseline ECG, borderline exercise ECG, LBBB \& RBBB, LVH, chronic digitalis administration, ventricular pacemakers, when patients are not suitable for exercise due to reduced exercise capacity like obesity, chronic lung diseases, peripheral vascular disease or skeletomuscular disorders, risk stratification before noncardiac surgical procedures in patients unable to exercise maximally, and for detection of viable myocardium. 